\title{
Interfacial capacitance immunosensing using interdigitated electrodes: effect of the insulation/immobilization chemistry
}

Received 00th January 20xx, Accepted 00th January 20xx

DOI: $10.1039 / x 0 x \times 00000 x$

\author{
F. Rafael. Castiello, ${ }^{\text {a James Porter, }}{ }^{\text {a Paresa Modarresa }}{ }^{\mathrm{a}}$ and Maryam Tabrizian*a,b
}

With the aim of improving the reproducibility of capacitive immunosensors, we performed a comparative study of four different insulating/immobilization chemistries. Each chemistry targeted different areas of an interdigitated electrode including an alkyl thiol monolayer on the electrode surface, an amino silane monolayer on the gaps between electrodes, and conformal coatings via passive adsorption of the probe and a spin-coated layer of poly(methyl methacrylate) (PMMA). We analyzed the dielectric properties of these chemistries by comparing their capacitive behavior through equivalent circuit modeling and correlate the observed behavior with their surface characteristics using atomic force microscopy and finite element modeling. We found that surface binding events occurring in the interdigitated electrode gaps play a major role in the overall change in capacitance. This was confirmed via finite element modeling showing an increased electric field intensity in the electrode gaps by $14 \%$, as compared to directly above the electrodes. Among the investigated surface chemistries, PMMA conformal coating produced a smooth surface (Rq roughness $=0.21 \pm 0.02 \mathrm{~nm}$ ) providing the most reproducible and stable capacitance change $(15.6 \pm 0.4 \%)$ in response to specific antigen-antibody binding.

\section{Introduction}

The most basic architecture of an immunosensor consists of a receptor, either antibody or antigen, and a transducer which is capable of detecting binding between the two ${ }^{1}$. A wide range of transduction mechanisms can be used for the development of immunosensors including: optical, electrical, mechanical and magnetic 2 . Among such transduction mechanisms, electrical analytical methods commonly present instrumental simplicity and high sensitivity. This has encouraged the miniaturization of electrical immunosensors in recent years, making them ideal for the growing field of point of care diagnostic devices ${ }^{3}$.

A particular case of electrical transductions methods is impedance sensing, which in essence measures changes in the electrical impedance of an electrode/electrolyte interface over a range of applied frequencies. In the absence of a redox probe (non-Faradic), the interfacial capacitance at the electrode/electrolyte interface can be used as a sensitive way of measuring binding events, hence making such sensors commonly known as capacitive immunosensors ${ }^{4}$. The absence of a redox probe in these types of sensors provides design and experimental advantages over its Faradic counterparts where a three-electrode system is required, depletion of the probe can occur and catalytic modification of the sensing interface is needed ${ }^{3}$. The most common kind of capacitive immunosensor

\footnotetext{
a. Biomedical Engineering Department, McGill University, Montreal, QC, Canada.

*E-mail: maryam.tabrizian@mcgill.ca

b. Faculty of Dentistry, McGill University, Montreal, QC, Canada.

†Electronic Supplementary Information (ESI) available: Microfabrication protoco for IDEs, additional characterization measurement, and typical impedance spectra change for capacitive detection of antigen-antibody binding of the different insulation/immobilization chemistries. See DOI: 10.1039/x0xx00000x
}

is based on coplanar interdigitated electrodes (IDEs) ${ }^{4}$. This type of electrodes provides a large sensing surface, and their performance can be adjusted by modifying their dimensions.

However, while practically simpler, relative unpredictability and limited reproducibility have made capacitive immunosensors unfavored compared to analogous Faradaic approaches ${ }^{3-5}$. A possible reason for this unpredictable response is difficulties associated with the production of compact and pinhole-free insulating/immobilization layers. Such defects can cause a "short circuit" on the capacitive layer, ultimately decreasing its sensitivity ${ }^{4}$. Some common insulating/immobilization strategies include self-assembled monolayers (SAM) of alkyl thiols ${ }^{6-9}$, polymers ${ }^{10-13}$, and silanes 14-16. The "short circuit" problem could be particularly troublesome when using a SAM of alkyl thiols on gold electrodes since small SAM defects can lead to large changes in capacitance or a complete loss of sensitivity $1,17,18$. Furthermore, a variety of architectures have been reported in literature investigating the covalent immobilization of biomolecules on different regions of the sensor such as on the electrodes 6-9, 19, in the gaps between electrodes ${ }^{14-16,20}$ and a conformal coating covering both 10,11, 21, 22. However, so far, no systematic studies have been performed to evaluate the effect of these architectures on the sensor performance. This suggests the need for further research in probe immobilization chemistry and architecture which help to improve reproducibility ${ }^{5}$.

In this work, we aim to investigate the insulation/immobilization chemistry and architecture that renders the largest capacitance change detectable for antigenantibody binding with the most reproducible results. To achieve this, we compared four different insulating/immobilization chemistries commonly found in literature, targeting different 
areas of a capacitive immunosensor which included: an alkyl thiol SAM on the electrode surface, an amino silane monolayer on the gaps between electrodes, conformal coatings via passive adsorption of the probe and a spin-coated layer of poly(methyl methacrylate) (PMMA). Insulin and its antibody was used as a representative immunosensing system due to the interest of the authors in pursuing detection of such hormone in complex matrices. First, we evaluated the quality of these insulation/immobilization layers through equivalent circuit analysis using the fractional value of the exponential coefficient of a constant phase element (CPE). Next, we correlated the obtained CPE fractional values to the surface characteristics of each chemistry using atomic force microscopy (AFM). Then, the different insulation/immobilization layers were further functionalized with antigen, and the capacitance change in response to antigen-antibody binding was evaluated. After selecting the most reproducible chemistry, we evaluated the effect of IDEs size on the sensor performance. Finally, two negative controls (PMMA-coated IDEs functionalized with a non-specific antigen, and with no antigen on their surface, respectively) were used to validate the optimized surface chemistry through assessing the specificity of the immunosensor via a secondary antibody assay.

\section{Materials and Methods}

Absolute ethanol and phosphate-buffered saline (PBS) 10X were purchased from Fisher Scientific (Fair Lawn, NJ, USA). Phosphate-buffered saline 1X (PBS) tablets and Tween 20 were purchased from BioShop Canada Inc. (Burlington, Ontario, Canada). N-(3-Dimethylaminopropyl)-N'-ethylcarbodiimide hydrochloride (EDC), N-Hydroxysuccinimide (NHS), Aminopropyl)triethoxysilane $99 \%$ (APTES), bovine serum albumin (BSA) and Hydrochloric acid $(\mathrm{HCl})$ were purchased from Sigma-Aldrich (St. Louis, MO, USA). 16-Mercaptohexadecanoic acid (MHDA) was purchased from ProChimia Surfaces Sp. (Zacisze, Sopot, Poland). Tris-buffered saline (TBS) with $1 \%$ Casein from BIO-RAD. Anti-insulin antibody $(6.2 \mathrm{mg} / \mathrm{mL})$ and human insulin were purchased from PROSPECT (Ness, Ziona, Israel). Anti-glucagon antibodies $(200 \mu \mathrm{g} / \mathrm{mL})$ were purchased from Santa Cruz Biotechnologies, Inc. (Mississauga, ON, Canada). Poly(methyl methacrylate) (PMMA 950 A2) was purchased from MicroChem Corp.

\section{Microfluidic handling system}

A microfluidic chamber was designed using a CAD software. Using a clear FLGPCLO2 resin (a mixture of methacrylate monomers and oligomers), the device was then constructed by 3D printing (Form2 printer from FormLabs) with a post-cured tensile strength of $65 \mathrm{MPa}$.

\section{Impedance spectra acquisition}

Impedance measurements were carried out using a 4294A Agilent precision impedance analyzer with a frequency range of $40 \mathrm{~Hz}-110 \mathrm{MHz}$. The impedance analyzer (Agilent 4294A) was calibrated for broadband $A C$ measurements in a 2-point circuit.
The impedance spectrum was obtained over the frequency window of $40 \mathrm{~Hz}-10 \mathrm{MHz}$, with no sensor, to account for effects of the circuit. Calibrations were done using an open circuit, a shorted circuit (source connected to drain), and under a constant load. The constant load was provided by a known standard resistance of 50 ohms. Interdigitated electrodes were connected through the fixture $16089 \mathrm{~B}$ and spring loaded pins to the analyzer. Impedance magnitude and phase angle were recorded performing frequency scans over the range of $40 \mathrm{~Hz}$ $10 \mathrm{MHz}$ with an oscillating AC potential of $10 \mathrm{mV}$ with $0 \mathrm{~V} \mathrm{DC}$ bias. All frequency scans were taken at bandwidth 5 (highest precision), and for all reported measurements, the spectrum was captured 3 times, 3 minutes apart and then averaged.

\section{Microscopy image acquisition}

Image acquisition was performed with an inverted microscope (Eclipse TE 2000-U, Nikon Corp, Mississauga, ON, Canada), with fluorescence capabilities. All images were captured using a CCD camera (Retiga-2000R, Q imaging, Surrey, BC, Canada) and Nikon NIS-Elements D software.

\section{Atomic Force Microscopy}

AFM surface analysis were performed using a Nanoscope III instrument (Digital Instruments, USA) and Nanoscope v 5.12r5 software. AFM images were acquired in tapping mode in air at room temperature with a silicon probe having a nominal spring constant of $42 \mathrm{~N} / \mathrm{m}$ and a nominal resonance frequency $330 \mathrm{kHz}$ (model PPP-NCHR, NANOSENSORSTM).

\section{Interdigitated electrode fabrication}

Three electrode sets of IDEs $5 \times 5,10 \times 10$ and $15 \times 15 \mu \mathrm{m}$ (width $(w) \times$ spacing (s) in size were fabricated in a cleanroom environment. The IDEs fingers length was $1 \mathrm{~mm}$, and a total surface area of $0.5 \mathrm{~mm}^{2}$ was kept constant by varying the total number of fingers when changing the electrode finger width and spacing. Glass wafers $1 \mathrm{~mm}$ thick and $127 \mathrm{~mm}$ in diameter were used as substrates. The wafers were cleaned with acetone under sonication for $5 \mathrm{~min}$, rinsed with isopropyl alcohol (IPA), distilled water and dried with $\mathrm{N}_{2}$. The substrate was then heated to $100^{\circ} \mathrm{C}$ in a YES priming oven under vacuum for $5 \mathrm{~min}$ to evaporate any leftover solvents and then cooled with a steam of $\mathrm{N}_{2}$. Next, LOR5B ${ }^{\mathrm{TM}}$ (Microchem, Newton, MA) was spincoated at $1000 \mathrm{rpm}$ for $45 \mathrm{~s}$ onto the glass substrate and baked at $180^{\circ} \mathrm{C}$ for $5 \mathrm{~min}$. After allowing the wafer to cool down, Microposit $^{\mathrm{TM}} \mathrm{S} 1813^{\mathrm{TM}}$ (Shipley, Marlborough, MA) was spincoated at $4000 \mathrm{rpm}$ for $30 \mathrm{~s}$ and baked at $115^{\circ} \mathrm{C}$ for $1 \mathrm{~min}$. The substrate was then exposed to UV light with a dose of 136 $\mathrm{mJ} / \mathrm{cm}^{2}$ under a chrome photomask. Finally, the substrate was developed by immersing the substrate in Microposit $\mathrm{MF}^{\circledR}-319$ (Shipley) developer for $70 \mathrm{~s}$ without agitation and immediately washed with deionized water and dried with N2. A $10 \mathrm{~nm}$ titanium adhesion layer and a $50 \mathrm{~nm}$ gold layer were deposited using a NexDep E-beam evaporator (Angstrom Engineering Inc). The substrate was then immersed in Microposit Remover 1165 (Shipley) at $70^{\circ} \mathrm{C}$ under sonication to lift off the metal layer and reveal the patterned electrodes. Finally, the wafer was diced 
using a diamond saw (Disco DAD3240) into individual sensor chips $(20 \mathrm{~cm} \times 19 \mathrm{~cm})$.

\section{Immobilization chemistry}

Prior to use, all IDEs were cleaned with acetone under sonication for $5 \mathrm{~min}$ then rinsed with IPA, distilled water, and dried with a stream of $\mathrm{N}_{2}$. Additionally, Kapton stickers were placed on the IDEs connection pads to protect them from surface functionalization which could hinder their conductivity.

\section{Poly(methyl methacrylate) (PMMA) coating and functionalization}

The PMMA functionalization of the IDEs was adopted from a protocol reported by $\mathrm{K}$. Kamgil et al. 23 with minor modifications. First, commercially obtained PMMA was spincoated undiluted onto IDEs at $5000 \mathrm{rpm}$ for $45 \mathrm{~s}$ and baked at $180{ }^{\circ} \mathrm{C}$ for $90 \mathrm{~s}$, obtaining a final thickness of $66 \mathrm{~nm}$, measured by ellipsometry. Then, the IDEs were treated with $\mathrm{O} 2$ plasma at $100 \mathrm{~W}$ for $150 \mathrm{~s}$ to create hydroxyl groups on its surface and immediately immersed in a $10 \%(\mathrm{v} / \mathrm{v})$ solution of APTES in MQ water at $70^{\circ} \mathrm{C}$ for $5 \mathrm{~min}$. Finally, they were quenched in ethanol and baked at $110^{\circ} \mathrm{C}$ for $10 \mathrm{~min}$.

\section{Alkyl thiol self-assembly monolayer (SAM) functionalization}

IDEs were immersed in an ethanolic solution of $2 \mathrm{mM}$ MHDA overnight $(\approx 18 \mathrm{~h})$ at room temperature to allow self-assembly monolayer (SAM) formation. The substrates were then thoroughly rinsed with absolute ethanol, DI water and dried under a stream of $\mathrm{N}_{2}$.

\section{(3-Aminopropyl)triethoxysilane (APTES) functionalization on IDEs gaps}

IDEs were treated with $\mathrm{O}_{2}$ plasma at $100 \mathrm{~W}$ for $150 \mathrm{~s}$ and immediately immersed in a $10 \%(\mathrm{v} / \mathrm{v})$ solution of APTES in MQ water (Barnstead, $18.2 \mathrm{M} \Omega$ distilled water) at $70{ }^{\circ} \mathrm{C}$ for $5 \mathrm{~min}$. The chip was then quenched in ethanol, rinsed with $M Q$ water, dried with $\mathrm{N}_{2}$ and baked at $110^{\circ} \mathrm{C}$ for $10 \mathrm{~min}$. The APTES form a self-assembly layer directly to the glass, which leads to functionalization of the gaps only, leaving the gold surfaces bare.

\section{Passive adsorption (PA) functionalization}

The PA functionalization of the IDEs was performed following a protocol reported by $\mathrm{H}$. Cui et al. ${ }^{24}$ with few modifications. First, the IDEs were treated with $\mathrm{O} 2$ plasma at $50 \mathrm{~W}$ for $30 \mathrm{~s}$ to make the electrode surface hydrophilic. Immediately after, the chip was immersed in a solution containing $1 \mathrm{mg} / \mathrm{mL}$ insulin in PBS and incubated at $4{ }^{\circ} \mathrm{C}$ overnight. Then, the chip was rinsed with copious amounts of PBS and the surface blocked by immersion in a solution containing $5 \%(\mathrm{v} / \mathrm{v})$ BSA, $1 \%(\mathrm{v} / \mathrm{v})$ Casein and $0.05 \%(v / v)$ Tween-20 in TBS buffer (BCB buffer) for $30 \mathrm{~min}$.

\section{Covalent immobilization of insulin}

After SAM, APTES and PMMA functionalization, the chips were loaded into the microfluidic chamber for the remaining steps of insulin immobilization. Figure 2 shows a schematic summary of all peptide immobilization chemistries performed.

For IDEs functionalized with PMMA and APTES, $5 \mathrm{mg} / \mathrm{mL}$ NHS and $2 \mathrm{mg} / \mathrm{mL}$ EDC were mixed in MES buffer ( $\mathrm{pH}$ 6.0). Then, $125 \mu \mathrm{L}$ of the NHS/EDC mixture was added to $100 \mu \mathrm{L}$ of an aqueous solution of insulin with a concentration of $1 \mathrm{mg} / \mathrm{mL}$. After reacting for $15 \mathrm{~min}, 825 \mu \mathrm{L}$ of PBS $10 \mathrm{X}$ was added to the mixture to raise its $\mathrm{pH}$ to 7.4 and immediately injected into the system. Finally, the mixture was incubated for $1 \mathrm{~h}$, allowing the covalent bonding between carboxyl groups on insulin and amine groups on APTES to occur.

For IDEs functionalized with alkyl thiol SAM, the NHS/EDC mixture in MES buffer was first injected into the chamber allowing it to react for $15 \mathrm{~min}$. Then, a PBS solution of insulin with a concentration of $1 \mathrm{mg} / \mathrm{mL}$ was injected into the chamber and allowed to react for $1 \mathrm{~h}$ to form a covalent bond between carboxyl groups on the SAM and the amine groups on the insulin.

After insulin incubation, all surfaces were rinsed by flowing $1 \mathrm{~mL}$ of PBS (1X) with $0.05 \%$ Tween-20 (PBS-T) through the chamber for $10 \mathrm{~min}$. Next, any remaining reactive sites and nonspecific binding sites on the surface were blocked by injecting $B C B$ buffer and incubating for $30 \mathrm{~min}$. A final rinsing step was performed using $1 \mathrm{~mL}$ of PBS-T.

\section{Antigen-antibody binding detection}

Insulin monoclonal antibody was injected into the system with a final concentration of $10 \mu \mathrm{g} / \mathrm{mL}$. Once the solution reached the sensing chamber, the flow was stopped and the antibody was incubated for $1 \mathrm{~h}$. Then, the surface was rinsed with $1 \mathrm{~mL}$ of PBS-T for $10 \mathrm{~min}$. After rinsing, the system was allowed to equilibrate for at least $60 \mathrm{~s}$, and then the impedance spectrum was captured. The impedance spectra from the antigenfunctionalized and blocked surface were used as a baseline, to determine the capacitance change caused by antibody binding. To assess the assay specificity and as an additional way to evaluate the sensor functionalization, a secondary fluorescence antibody (Alexa Fluor 647 goat anti-mouse) was injected into the system with a final concentration of $20 \mu \mathrm{g} / \mathrm{mL}$ and incubated for $1 \mathrm{~h}$. Then, the surface was rinsed with $1 \mathrm{~mL}$ of PBS$\mathrm{T}$ for $10 \mathrm{~min}$, the system was allowed to equilibrate for at least 60 s, and the impedance spectrum was recorded. As with the primary antibody, the impedance spectra from the antigenfunctionalized and blocked surface were used as a baseline, to determine the total capacitance change caused by secondary antibody binding.

\section{Statistics}

For all impedance spectra analysis, 3 independent experiments were performed, where the experimental data was fitted to an equivalent circuit represented by equation 5 using OriginLab 2018 (b.9.5.5.409) with a confidence interval of 95\%. Then, the mean value of these circuit elements was calculated and is presented in the manuscript as mean \pm standard deviation (SD). Similarly, for AFM analysis the data is express as the mean of at least 3 independent experiments \pm SD. Means that are statistically different are indicated with a subscript asterisk (*). 

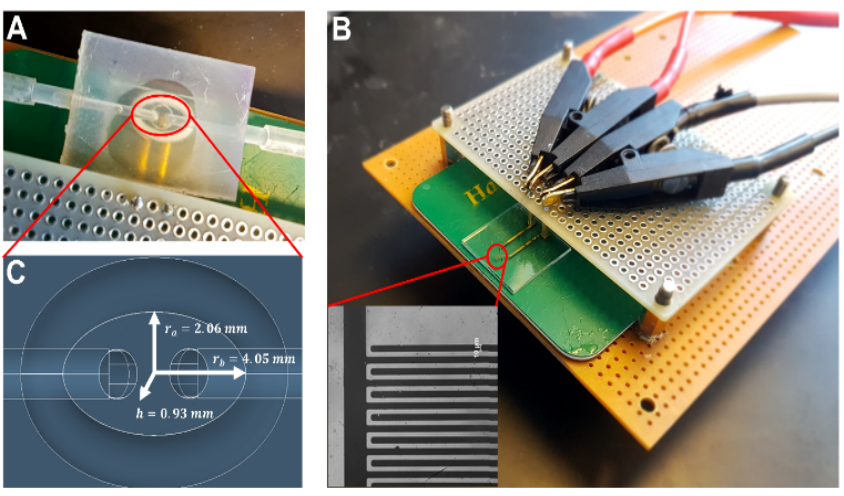

Fig. 1 Experimental setup for impedance measurements: A) the microfluidic chamber, B) the electrical connection between the IDEs and to the impedance analyzer and C) the schematic representation of the 3D printed chamber showing its dimensions with a total volume of $25 \mu \mathrm{L}$.

Microsoft Excel 2016 was used to perform the One-way ANOVA test followed by Bonferroni's test correction to evaluate the statistical difference of multiple samples, where $\mathrm{P}<0.05$ was considered a significant difference.

\section{Results and Discussion}

\section{Microfluidic device fabrication and experimental setup}

For stable and consistent measurements the sensor was interfaced with the analytes using a microfluidic chamber constructed via 3D printing. The microfluidic device consists of an inlet and outlet of $1 \mathrm{~mm}$ in diameter and an elliptical chamber with a height of $0.93 \mathrm{~mm}$, a small radius ( $\mathrm{ra}$ ) of 2.06 $\mathrm{mm}$, and large radius (rb) $4.05 \mathrm{~mm}$, with a total volume of approximately $25 \mu \mathrm{L}$. Figure 1 shows the final experimental setup that includes a microfluidic chamber (Fig.1 A) and the electrical connection between the IDEs and the impedance analyzer (Fig.1 B). Figure 1C displays a schematic presentation of the dimension of the microfluidic chamber.

\section{Insulation/immobilization chemistries}

One of the crucial aspects affecting the reproducibility of capacitive immunosensors is the appropriate design of the sensor surface. The surface morphology of the insulating layer needs to be homogeneous and densely packed to prevent a decrease in the sensor's sensitivity due to surface irregularities such as pinholes $4,25-27$. Therefore, we investigated the effect of four of the most commonly encountered insulating/immobilization strategies in literature for capacitive sensing. In capacitive sensing, the binding of a molecule is expected to produce a change in thickness on the dielectric double layer directly proportional to the molecule size ${ }^{28}$. For this reason, insulin ( $\mathrm{MW}=5807.57 \mathrm{Da}$ ) was immobilized onto the IDEs surface, and sensing was performed by detecting attachment of the much larger insulin antibody (MW=150kDa).

Four of the more commonly reported surface chemistries and architectures targeting different areas on the surface of the sensor were chosen: a conformal functionalization with PMMA that covers the entire sensing area (Fig. 2A), a SAM using 16mercaptohexadecanoic acid covering only the electrodes (Fig. 2B), a layer of (3-aminopropyl)triethoxysilane (APTES) covering the space between the electrodes (Fig. 2C) and the passive adsorption (PA) of the antigen covering both electrodes and spaces (Fig. 2D).

\section{Equivalent circuit model fitting}

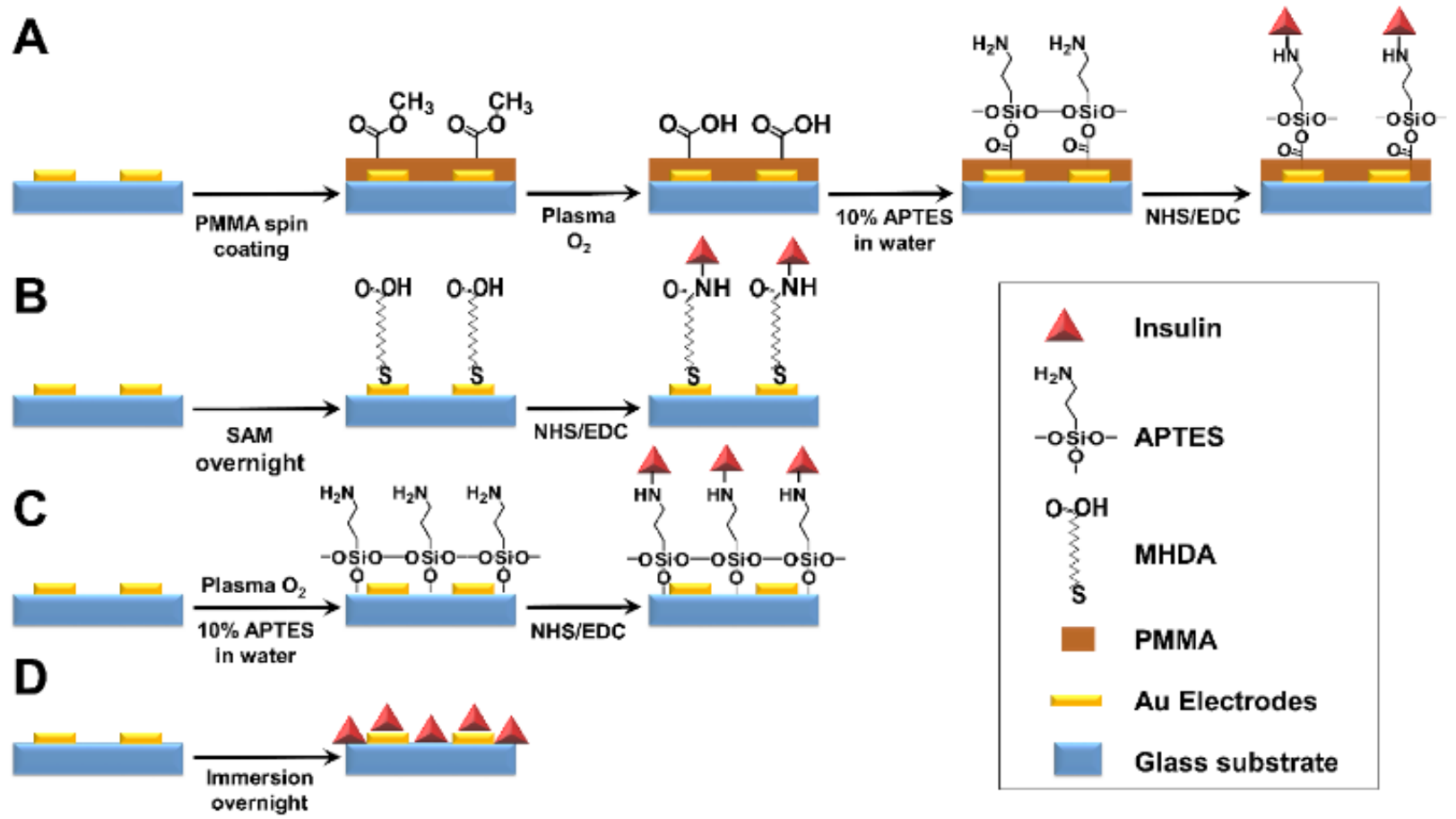

Fig. 2 Schematic representation of the different insulin immobilization chemistries and architectures used on IDEs. A) PMMA, B) SAM, C) APTES, D) PA. 

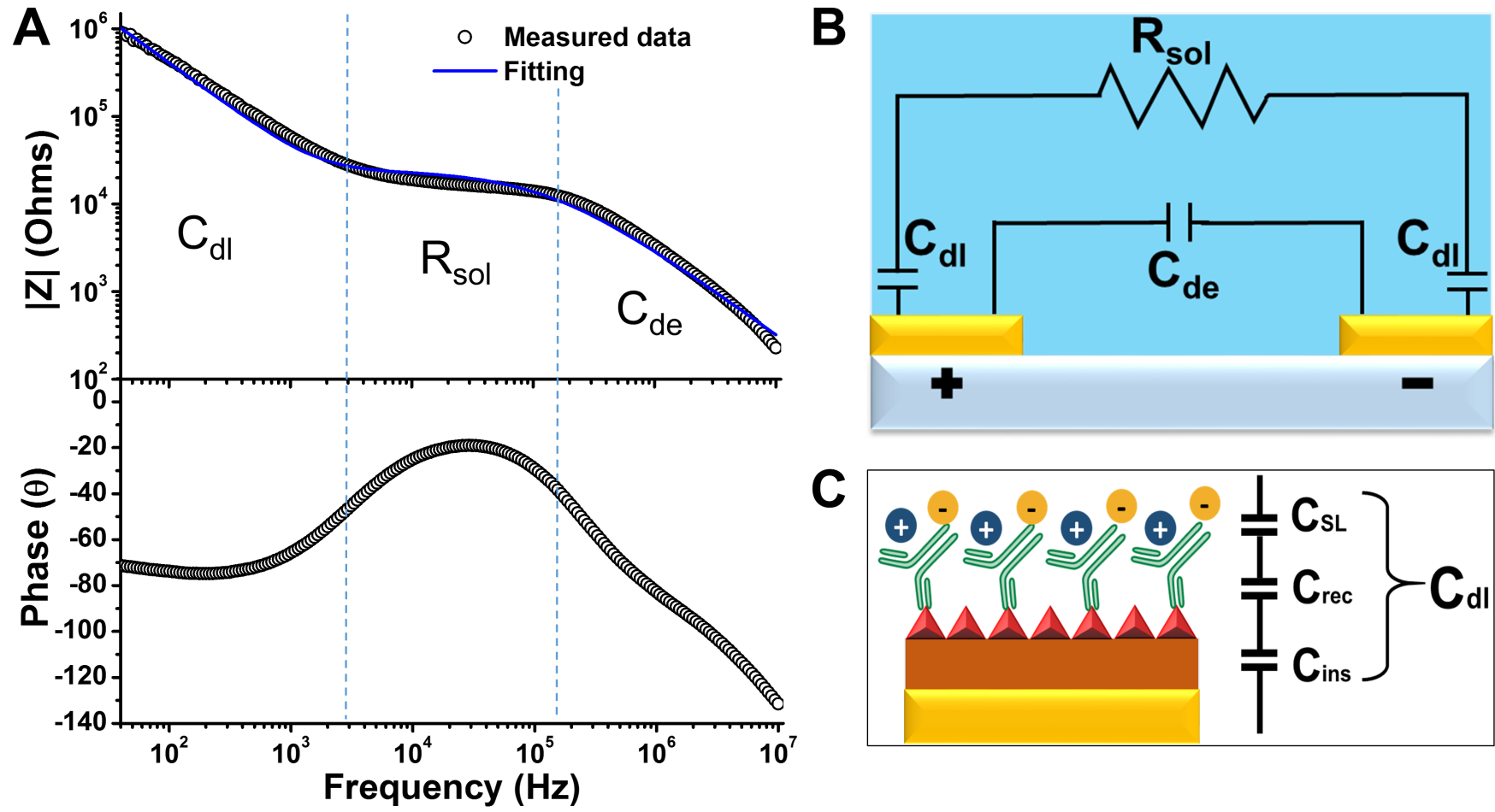

Fig. $3 \mathrm{~A}$ ) Interfacial impedance and phase angle spectra of IDEs in the absence of a redox probe in MQ water. $C_{d l}$ corresponds to the double layer capacitance, $R_{\text {sol }}$ to the solution resistance and $C_{d e}$ to the dielectric capacitance of the media. B) Schematic representation of the equivalent circuit model used for fitting measured data in $A$. C) Schematic representation of a model of capacitors in series that define the double layer capacitance for a typical capacitive biosensor with an electrode-solution interface. $C_{\text {ins }}$ corresponds to an insulating/immobilization layer. $\mathrm{C}_{\mathrm{rec}}$ includes the contribution of the immobilized antigen layer, any additional blocking proteins, and the specific antibody binding layer. $\mathrm{C}_{\mathrm{SL}}$ represents the final Stern Layer.

The equivalent circuit representation is commonly used in literature as a powerful tool that allows the characterization of an electrode-electrolyte system 25, 29. This representation approximates the experimental impedance data with ideal impedance elements arranged in series and/or in parallel 25, 29. In this manner, the change of impedance in one element such as a resistance or a capacitance can be evaluated by correlating the overall impedance change to a physical phenomenon such as antigen-antibody binding.

The spectra of the interfacial impedance and phase angle of an IDEs in the absence of a redox probe (non-Faradaic regime) and in aqueous media is shown in Figure $\mathbf{3 A}$ 30, 31. The total impedance of this system is typically represented by the equivalent circuit depicted in Figure 3B ${ }^{6,30}$. It is observed that there are three clearly defined zones in the spectra (Fig. 3A), corresponding to the three elements in the equivalent circuit that include the two parallel branches (Fig. 3B). Then, the total impedance of the system $(Z)$ as a function of frequency $(f)$ is expressed as:

$$
\frac{1}{|\mathrm{Z}|}=\frac{1}{\sqrt{R_{S o l}^{2}+\frac{1}{\left(\pi f C_{d l}\right)^{2}}}}+2 \pi f C_{d e}
$$

For frequency ranges below $10^{5} \mathrm{~Hz}$ (Fig. 3A), current does not flow through the dielectric capacitor $\left(C_{d e}\right)$, leaving it inactive ${ }^{31}$.
In this case, the total impedance of the system corresponds only to the double layer capacitance $\left(C_{d l}\right)$ and solution resistance $\left(R_{\text {sol }}\right)$ in series. The $\mathrm{R}_{\text {sol }}$ region can be distinctly observed by a peak in the phase angle signal corresponding to the plateau of the impedance magnitude signal ${ }^{32}$. In this case, the total impedance of the system simplifies to:

$$
|\mathrm{Z}|=\sqrt{R_{\text {Sol }}^{2}+\frac{1}{\left(\pi f C_{d l}\right)^{2}}}
$$

Since the solution composition is usually constant during experiments, in essence, the system reports changes on the interfacial capacitance. The interfacial capacitance can be represented by a model of various capacitive layers in series ${ }^{4}$. Figure $\mathbf{3 C}$ shows a schematic representation of such capacitors in series which define the total capacitance of the biosensor. $C_{\text {ins }}$ corresponds to an insulating/immobilization layer. $C_{\text {rec }}$ includes the contribution of the immobilized antigen layer, any additional blocking proteins, and the specific antibody recognition layer. $C_{S L}$ represents the final Stern Layer. Thus, during biosensing, the antigen-antibody binding is responsible for the overall change in the total capacitance. The total capacitance of the double layer $\left(C_{d l}\right)$ is then expressed as: 

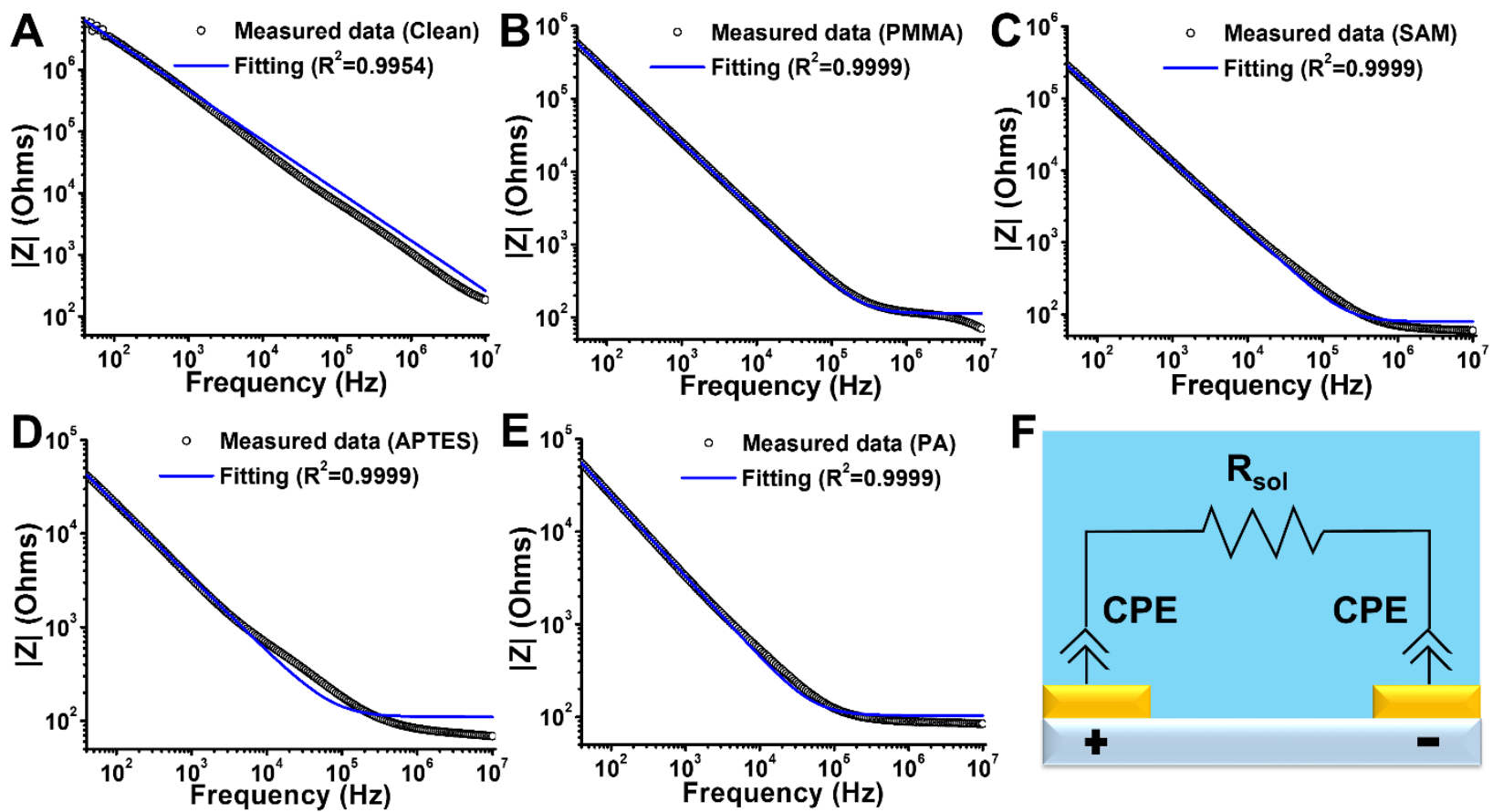

Fig. 4 Representative impedance spectra and fitted curves in PBS 1x between the frequency range of $40 \mathrm{~Hz}-10 \mathrm{MHz}$ for $10 \times 10 \mu \mathrm{m}$ IDEs: A) clean, B) spin-coated PMMA, C) a SAM of alkyl thiol, D) a self-assembled layer of APTES in the space between the IDEs fingers, and E) a passively adsorbed (PA) layer of insulin and BCB buffer blocking. F) Schematic representation of the equivalent circuit model used for the fitting.

$$
\frac{1}{C_{d l}}=\frac{1}{C_{i n s}}+\frac{1}{C_{r e c}}+\frac{1}{C_{S L}}
$$

Overall, the model shows that the total capacitance in the system is governed by the smallest capacitance of the contributing layers. Additionally, equation 3 assumes an ideal insulation/immobilization and recognition layers where the effect of holes and other chemical heterogeneities are neglected ${ }^{4}$. This emphasizes the fact that the careful design and implementation of insulating layers is a crucial step for the development of capacitive immunosensors ${ }^{33}$.

Moreover, it has been empirically demonstrated that the capacitive behavior from the formation of an ionic double layer in solid electrodes does not behave ideally ${ }^{34}$. Instead, it has been observed that the double layer capacitance has a functional form that is frequency dependent ${ }^{35}$. This functional form is commonly known as a constant phase element (CPE), defined as:

$$
C P E=\frac{1}{(j \pi f)^{n} Q}
$$

Here, $j$ is the imaginary unit and $Q$ is equivalent to the capacitance $\left(C_{d l}\right)$ of a perfect capacitor. The coefficient $n$ of the CPE varies between 0 and 1, where $n=1$ (Eq. 4) represents the impedance of an ideal capacitor where $Q$ has units of capacitance $(F)$; otherwise $(n<1), Q$ has units of $F / s^{(1-n)}$. Therefore, the experimental impedance data can be fitted to the following equation:

$$
|Z|=\sqrt{R_{\text {Sol }}^{2}+\frac{1}{\left[(\pi f)^{n} Q\right]^{2}}}
$$

The fractional value of the CPE coefficient $n$ has been shown to account for a variety of the non-ideal conditions present in the capacitive sensing system, such as surface irregularities, chemical heterogeneities, and uneven ion adsorption onto the electrode surface ${ }^{35-37}$. Thus, the $n$ coefficient can represent a powerful parameter to assess the quality of the insulation/immobilization layer during the design of capacitive immunosensors.

To validate the application of the proposed CPE circuit model (Eq. 5), the impedance spectra of the electrode/electrolyte interface was recorded for a clean IDEs chip (Fig. 4A) and for IDEs functionalized with the four insulating/immobilization chemistries described in the previous section (Fig. 4B-E). For this analysis, IDEs chips with a $10 \times 10$ $\mu \mathrm{m}$ (width (w) $\times$ spacing (s)) were used. After functionalization, the chips were placed in the microfluidic chamber and the impedance spectra were recorded in PBS (1x). 

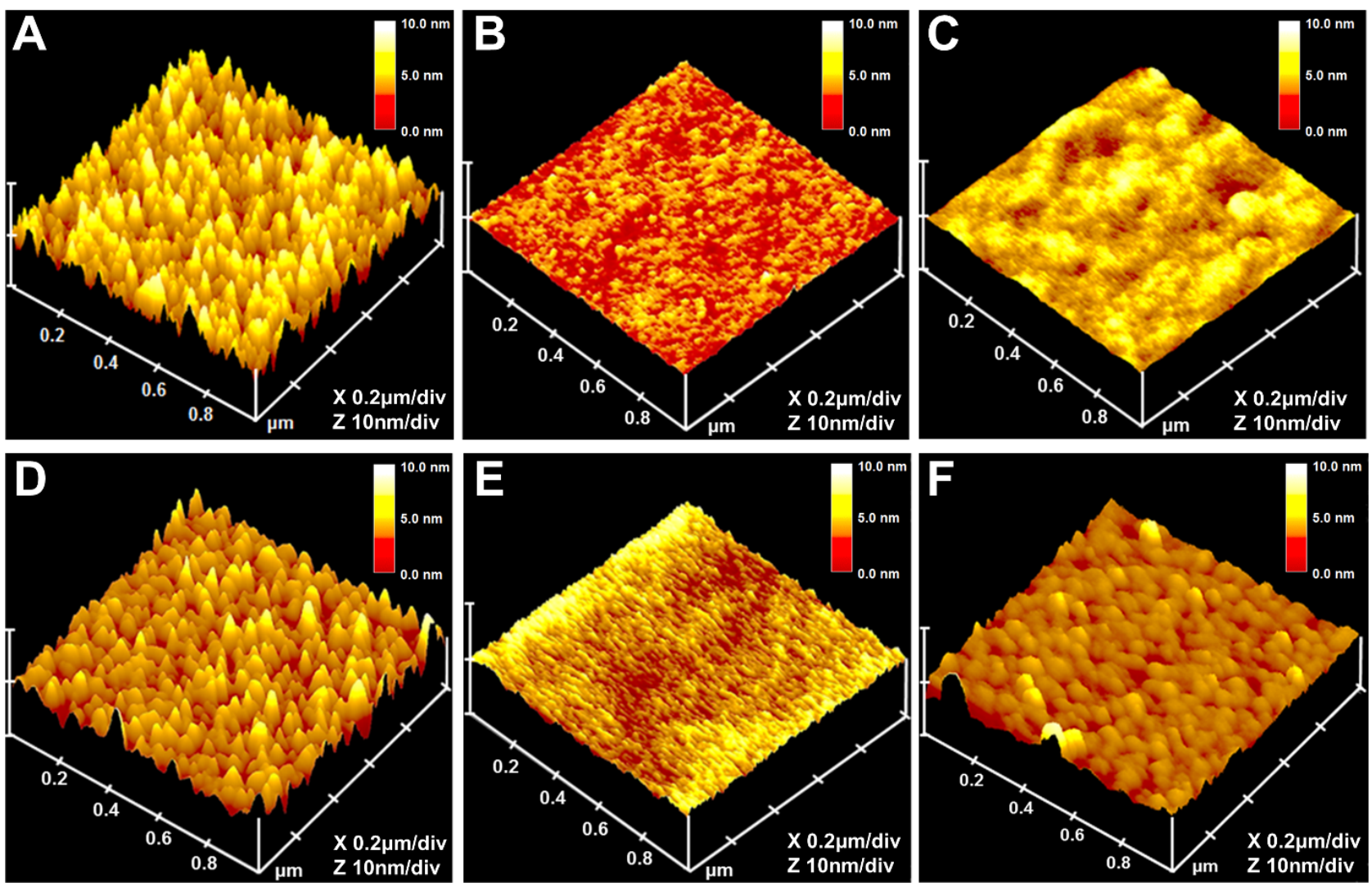

Fig. 5 Representative 3D images of a $1 \mu \mathrm{m}^{2}$ area of the IDEs sensing surface for: A) a clean Au electrode, B) clean glass in the gaps between electrodes, C) spin-coated PMMA on a gold electrode, D) MHDA SAM on a gold electrode, E) APTES on the IDEs gaps and F) PA of antigens on top of a gold electrode.

Table 1 Equivalent circuit parameters obtained through curve-fitting the experimental data with the equivalent circuit in Fig. 1B using Eq. 5. For this analysis 3 independent experiments were performed where a fitting was accomplished with a confidence interval of $95 \%$. All reported values correspond to the mean \pm standard deviation (SD) of the circuit element obtained from each independent fitting.

\begin{tabular}{|c|c|c|c|}
\hline Surface & CPE $n$ coefficient & Rsol $(\Omega)$ & $\boldsymbol{Q}\left(\mathbf{n F} / \mathbf{s}^{(1-n)}\right)$ \\
\hline Au clean & $0.90 \pm 0.05$ & $42 \pm 0.2$ & $3 \pm 1.5$ \\
\hline APTES & $0.83 \pm 0.02$ & $91 \pm 13.0$ & $233 \pm 36$ \\
\hline SAM & $0.95 \pm 0.01$ & $81 \pm 3.0$ & $32 \pm 6.0$ \\
\hline PMMA & $0.97 \pm 0.005$ & $111 \pm 2.0$ & $15 \pm 0.3$ \\
\hline PA & $0.91 \pm 0.46$ & $138 \pm 32$ & $238 \pm 43$ \\
\hline
\end{tabular}

According to the analysis and measurements from the fitted curves shown in Figure $\mathbf{4}$ and the extracted circuit parameters provided in Table 1, one can notice that for the working frequency range, the Cde term coming from the dielectric properties of the PBS $1 x$ solution can be neglected for all insulation/immobilization chemistries. It is also inferred that the behavior of the IDEs is predominantly capacitive for all insulating/immobilization chemistries in the frequency range between 0.1 to $1 \mathrm{MHz}$. On the other hand, the CPE $n$ coefficient of clean electrodes was found to be around 0.9 (Table 1) showing good agreement with previously reported data for experiments performed under similar conditions $32,38,39$. The insulation/immobilization chemistries that showed the largest deviation from ideal capacitive behavior were PA and APTES, while the ones showing the best capacitive behavior were SAM and PMMA. Additionally, PA presented the highest standard deviation values for the CPE $n$ coefficient from all surfaces while PMMA exhibited the lowest. Noteworthy, APTES showed a large change in capacitance $(Q)$ despite only modifying the gaps between electrodes. This is an interesting observation since little experimental research has been conducted regarding the optimal position for the recognition elements immobilization. To understand this phenomenon, further experiments were carried out and the results are reported in the following sections.

Morphological characterization of the insulating/immobilization layer

AFM was used to characterize the surface morphology of the different insulating/immobilization chemistries. Figure 5 shows a representative 3D image of a $1 \mu \mathrm{m}^{2}$ area of two controls (a clean electrode and clean glass in the gaps between electrodes) (Fig.5 A-B) and coated IDEs sensing surfaces as described in the previous section (Fig.5 C-F). The images are presented using the same $X Y$ scale and a vertical scale ranging from 0 to $10 \mathrm{~nm}$ (deep red to white) to allow proper comparison of the surface morphology among the different insulating/immobilization chemistries. Changes in surface features from the clean Au and glass surfaces confirmed the successful deposition of the different insulation/immobilization layers. For all functionalization strategies, a significant change in surface roughness expressed by root mean square roughness ( $\mathrm{Rq}$ ) in Table 2 was observed. The largest change in surface roughness was found for the PMMA coated surfaces. The large decrease of Rq was consistent with other published works where deposited 

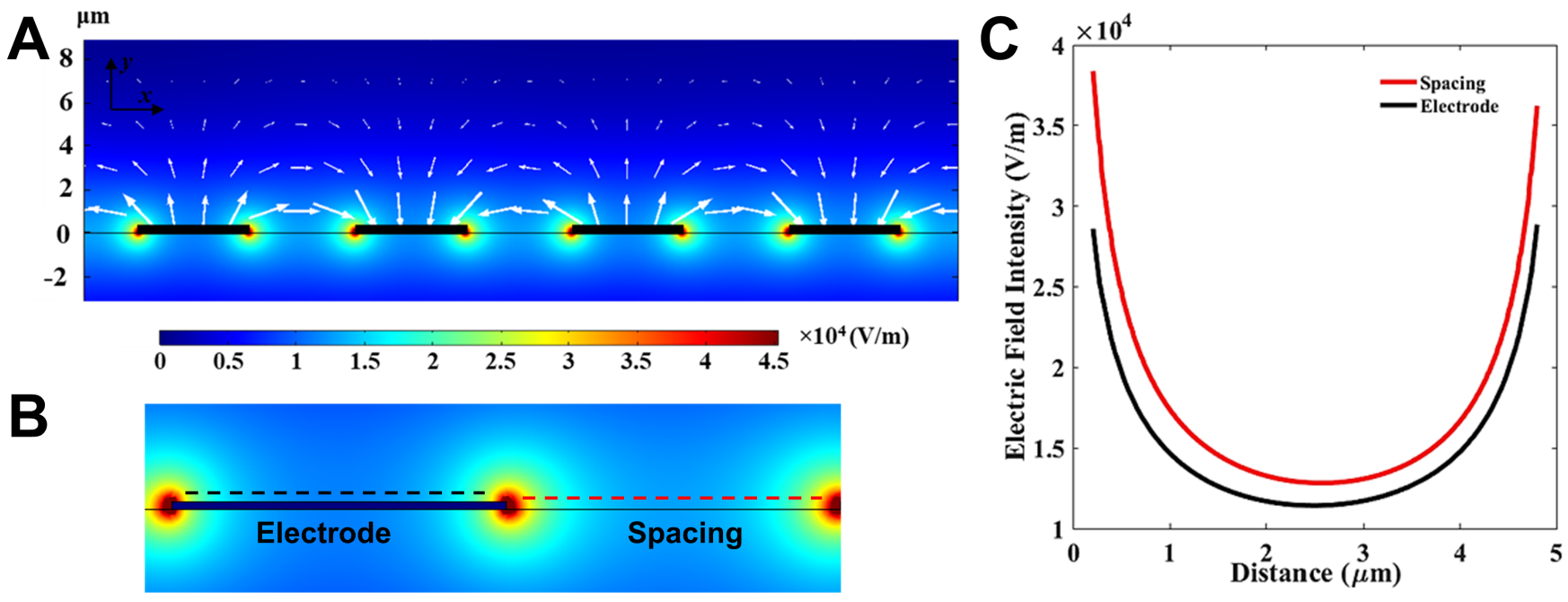

Fig. 6 A) 2-D cross section simulation of electric field intensity along the longitudinal axis of the chamber (x-direction). B) Image showing the enlarged electric field intensity around a single electrode and the gap using the same colour scale as A. C) Electric field intensity values at $80 \mathrm{~nm}$ above electrode and spacing surfaces (corresposnding to dotted lines on image B).

PMMA films significantly changed surface morphology and smoothed out rough surfaces 40,41 . The PA antigens-modified surfaces seemed to provide the most heterogeneous surface as the Rq measurements presented the largest standard deviation. This large standard deviation is likely due to the formation of aggregates in some areas of the surface, as clearly observable in Figure $\mathbf{S 2}$ provided in supporting information.

Table 2 AFM root mean square roughness ( $\mathrm{Rq}$ ) data from three independent samples $(n=3)$ of the different functionalization chemistries on IDEs. The data is presented as mean \pm SD of at least 3 independent experiments.

\begin{tabular}{|c|c|}
\hline Surface & Image Rq (nm) \\
\hline Au clean & $1.11 \pm 0.03$ \\
\hline Glass clean & $0.23 \pm 0.01$ \\
\hline APTES & $0.19 \pm 0.01$ \\
\hline SAM & $0.90 \pm 0.04$ \\
\hline PMMA & $0.21 \pm 0.02$ \\
\hline PA & $1.39 \pm 0.13$ \\
\hline
\end{tabular}

The AFM surface analysis matches well with the trend observed for the CPE $n$ coefficient analysis. Since the surface roughness is expected to have a significant impact on the capacitive behavior, we observed that the non-ideal capacitive behavior decreases with the decrease in surface roughness. Indeed, the IDEs coated with PMMA provided a small $\mathrm{Rq}$ of $0.21 \mathrm{~nm}$ and an $n$ coefficient as high as 0.97 . Conversely, the IDEs prepared with PA antigens presented an Rq of 1.39 and an $n$ coefficient of 0.9 . Additionally, the large standard deviation in the PA CPE $n$ coefficient measurement was consistent with that observed for Rq. Surprisingly, while the Rq for the APTES-coated IDEs was comparable to that of the clean glass, a noticeable decrease in its CPE $n$ coefficient was observed. A possible explanation could be that the APTES chemistry targeting only the gaps between the electrodes, produced a heterogeneous surface morphology seen in Figure 5E. In this case, the principle of a current leakage passing through an insulating layer does not apply, since in this sensing layout only the space between the IDEs were functionalized. To have a plausible explanation for this effect, the electric field intensity of IDEs was evaluated using finite element modeling (FEM).

\section{FEM of the electric field intensity for IDEs.}

The electric field inside the chamber for IDEs with a $5 \mu \mathrm{m}$ width, $5 \mu \mathrm{m}$ spacing, and $100 \mathrm{~nm}$ height was simulated by FEM using COMSOL 5.2 software (Burlington, MA). The simulated 2-D cross section of the device included the glass substrate, gold electrodes, the fluid (PBS), and PDMS as the ceiling (Fig. 6A, PDMS is not shown). The simulation was performed using the Electric Current Module in frequency domain. The constituent equations included:

$$
\begin{gathered}
\nabla . J=Q \\
J=\left(\sigma+j \omega \varepsilon_{o} \varepsilon_{r}\right) E+J_{e} \\
E=-\nabla V
\end{gathered}
$$

where $J$ is the current density, $J_{e}$ is the externally generated current, $E$ is the electric field intensity, $V$ is the applied voltage, and $Q$ is the charge. Furthermore, $\varepsilon_{O}$ and $\varepsilon_{r}$ are the electrical permittivity of the free space and the medium, respectively. The symbol $\omega$ represents the angular frequency of the applied voltage. Current conservation and electric insulation conditions were applied to all domains and outer geometry boundaries, respectively. The fluid, electrodes, and insulating layer domains were meshed with 15058 triangular elements with a minimum element quality of 0.73 . 

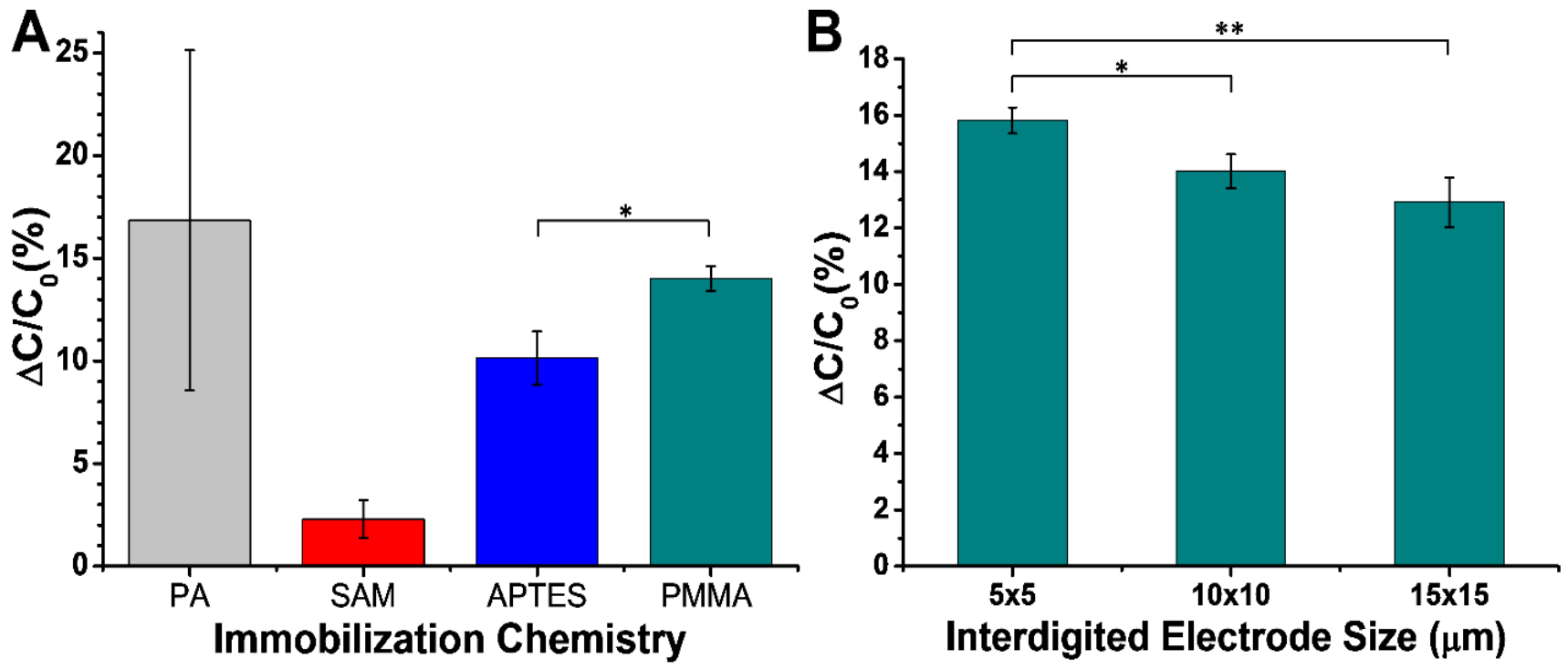

Fig. 7 Capacitance change for specific antibody binding represented as mean \pm SD calculated for three independent samples ( $n=3$ ) for: A) different immobilization/insulation chemistries, where the significant difference between APTES and PMMA chemistries was $\left({ }^{*}\right)$ P $<0.02$. B) different size of IDEs functionalized with PMMA, where the significant difference between $5 \times 5 \mu \mathrm{m}$ and $10 \times 10 \mu \mathrm{m}$ was $\left({ }^{*}\right) \mathrm{P}<0.02$ and $15 \times 15 \mu \mathrm{m}$ was $\left({ }^{* *}\right) \mathrm{P}<0.01$

The electrodes were biased with 0.1 volts at $100 \mathrm{~Hz}$ frequency and the electric field intensity values $(E)$ was calculated at $80 \mathrm{~nm}$ above electrode surfaces (black line) and above the spacing between electrodes (red line) (Fig. 6B). This distance was chosen as a distance at which the binding events are expected, taking into account the thickness of all the elements involved and the different immobilization chemistries used on the sensor surface.

The average electric field intensity within the spacing $(17.35$ $\mathrm{kV} / \mathrm{m}$ ) was found to be $14.3 \%$ higher than the average electric field intensity above electrode surfaces $(15.19 \mathrm{kV} / \mathrm{m})$. This could explain the CPE $n$ coefficient decrease and the large capacitance change after APTES functionalization of the IDEs spaces. Furthermore, the enhanced electric field intensity within the space separating two electrodes suggests that the events occurring in such spaces carry a significant weight in the overall change in capacitance. The FEM analysis is consistent with a previous report suggesting that the space between the electrodes significantly contributes to the total electric field intensity and current density distributions around the IDEs 42. Accordingly, it is important to consider the contribution of functionalized electrode spacing to the overall immunosensor performance in terms of sensitivity and reproducibility. As such, the insulating/immobilization chemistries that do not target the spacing between electrodes during functionalization would not profit from this enhanced electric field. Conversely, by not functionalizing the gaps between electrodes or properly blocking it, non-specific adsorption in this area could induce a large change in capacitance, ultimately reducing reproducibility.

\section{Capacitive immunosensing performance of the insulating/immobilization chemistries}

After depositing the different insulating/immobilization layers, covalent functionalization of the antigen was carried out. Since the measured impedance largely depends on the target size 4 , 17,25 , the small targeted antigen was immobilized on the surface and used as a probe for detection of the antibody in solution. With the exception of PA coating, the antigen was covalently bound to the insulating layer via NHS/EDC chemistry (Fig. 2). Then, all surfaces were blocked using buffer containing bovine serum albumin and casein to prevent non-specific binding.

Using the impedance spectra from the functionalized and blocked surfaces as the baseline, the change in capacitance after the antibody binding could be quantified as follows:

$$
\text { Capacitance change }=\frac{C_{B}-C_{0}}{C_{0}} * 100
$$

$C_{O}$ corresponds to the capacitance of the blocked surface and $C_{B}$ corresponds to the capacitance after binding. Figure 7A summarizes the capacitance change measured for the four surface chemistries. Figure $\mathbf{S 4}$ in supporting information presents the average impedance and phase spectra for of these chemistries from which the capacitance values were derived. As seen in this figure, the ratio of the capacitive to resistive term increases, the magnitude of the phase angle also increases and vice versa. However, since the resistive term is constant throughout each experiment, it can be inferred that the changes in phase angle is due to changes in the capacitive term. Therefore, the largest change in phase angle is seen at lower frequencies which directly corresponds to the largest shift in the impedance measurements.

Despite the large average change in capacitance, PA was the least reproducible sample, as indicated by a large standard deviation. This is probably caused by defects due to lack of covalent binding and heterogeneity on this surface, both of which also contribute to a much lower CPE $n$ coefficient. The SAM chemistry showed the smallest capacitance change 

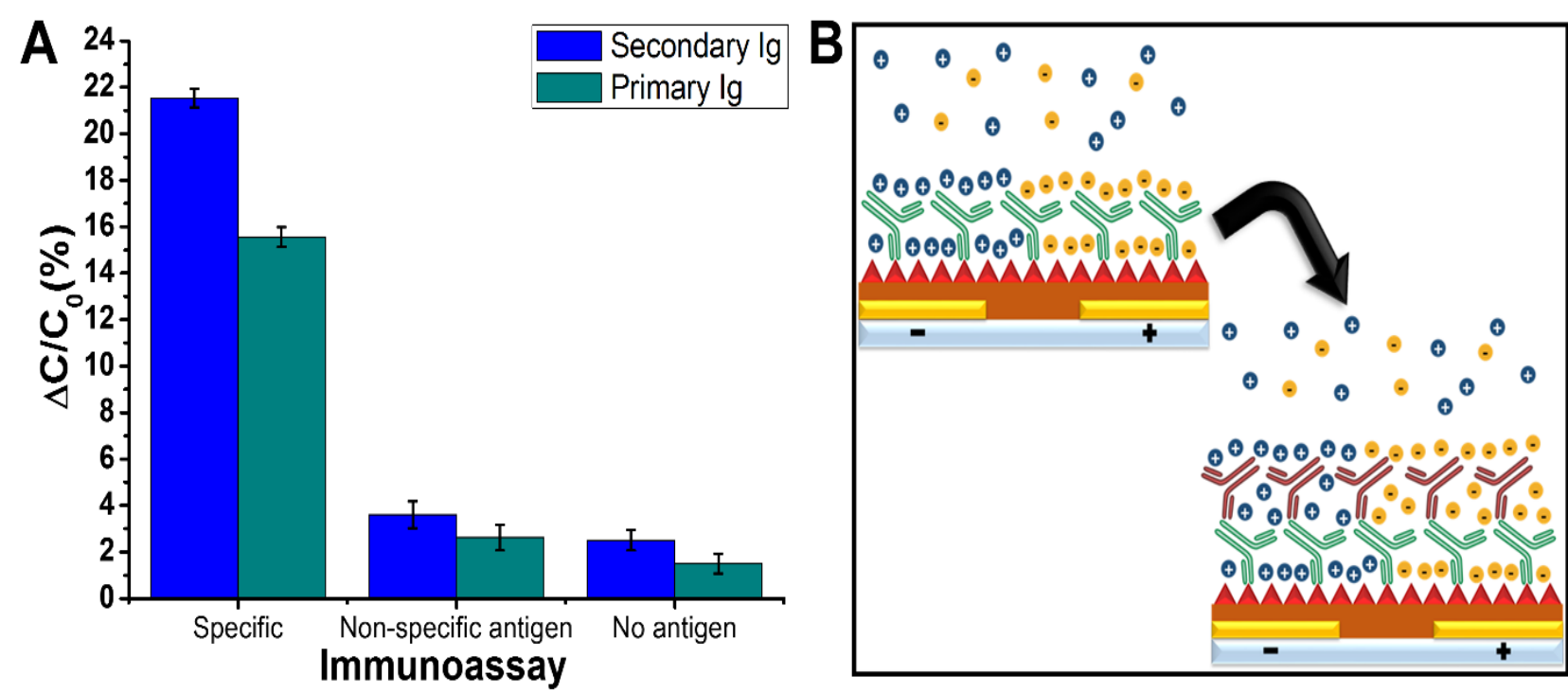

Fig. 8 A) Capacitance change for a specific assay and two negative controls consisting of a PMMA surface functionalized with a non-specific antigen and no antigen. B) Schematic representation of the specificity assay with a secondary antibody performed for this experiment.

despite having a homogeneous surface and an increase in the CPE $n$ coefficient. This poor performance might be associated with an interaction of the SAM with the Tween 20 used during the blocking and washing steps ${ }^{2}$. This was however necessary to avoid signal drifting during impedance measurement for all surface chemistries.

A one-way ANOVA test followed by Bonferroni's test correction showed that the change in capacitance for PA-functionalized IDEs was not significantly different from those functionalized with APTES and PMMA. Interestingly, APTES-functionalized IDEs showed a similar change in capacitance for antigen-antibody binding to that of the PMMA conformal coating despite only having the antigen immobilized in the space between electrodes. This can be explained by the increased electric field intensity in this region, shown by CPE $n$ coefficient analysis and FEM.

On the other hand, the capacitance change for PMMAfunctionalized IDEs proved to be significantly different from APTES $(P=0.02)$. Additionally, the capacitance change for PMMA-coated IDEs was reproducible and stable as this surface chemistry provided a compact and smooth surface (RMS roughness $=0.21 \pm 0.02 \mathrm{~nm}$ ), and a more ideal capacitive behavior (CPE $n$ coefficient $=0.97)$. These findings corroborate other reports on PMMA showing good thermal and mechanical stability, along with dielectric properties suited for metalinsulator applications ${ }^{43}$.

For the aforementioned reasons, PMMA-functionalized IDEs were further investigated regarding the effect of the electrode size on the sensor performance. Figure 7B shows the capacitance change for antigen-antibody binding for three different sizes of IDEs. In contrast to the large change in capacitance found among the different insulation/immobilization layers, the variation in the IDE sizes does not seem to greatly affect the sensor response. However, the capacitance change from $5 \times 5 \mu \mathrm{m}(w \times s)$ IDEs was slightly larger and presented a smaller standard deviation compared to $10 \times 10 \mu \mathrm{m}$ and $15 \times 15 \mu \mathrm{m}$ IDEs. This was expected since it has been shown that for IDEs, $80 \%$ of the electric field flows within half of the sum of the width and spacing of the electrode digits 44. This means that smaller electrodes are more specifically tuned to surface events and less sensitive to noise sources coming from the bulk of the solution ${ }^{44}$.

\section{Specificity of the capacitive immunosensor}

Finally, to assess the specificity of the PMMA capacitive immunosensors, we performed a specificity assay using a fluorescently labeled secondary antibody. Two negative controls were used consisting of PMMA coated IDEs functionalized with a non-specific antigen, and with no antigen on their surface, respectively. The results clearly indicated the specificity of the capacitive immunosensors using our approach (Fig. 8). Interestingly, when fluorescence imaging was performed to visually confirm the presence of the primary antibody in all surface, no fluorescence was detected for both controls (Fig. S5 provided in supporting information). This result could suggest that the capacitance immunosensing is more sensitive than fluorescence under our experimental conditions.

\section{Conclusions}

In this work, we assessed the insulation properties of four different chemistries by comparing the CPE $\mathrm{n}$ coefficient obtained through an equivalent circuit fitting. We found that spin-coated PMMA on IDEs presented the most ideal capacitive behavior (CPE $n$ coefficient $=0.97$ ) among the investigated surface chemistries due to the formation of a packed and smooth surface, as indicated by AFM analysis ( $R q=0.21$ ). Additionally, events occurring in the spacing areas between the IDEs showed to carry a significant weight in the overall change in capacitance. This was confirmed by: 1) the large change in capacitance for the APTES functionalization of the spacing area, 2) FEM simulations showing a $14 \%$ increase in the electric field intensity in IDEs spacing area compared to that on top of the 
electrodes and 3) a similar capacitance change in response to antigen-antibody binding to that of conformal coatings, despite having only half of the surface area functionalized. Comparing the capacitance change in response to antigen-antibody binding among chemistries, spin-coated PMMA IDEs produced the most reproducible and stable capacitance change. Additionally, we found that decreasing the IDEs size makes the system less sensitive to noise sources coming from the bulk of the solution, improving reproducibility. Overall, our finding suggests that polymeric conformal coatings are the most suitable candidates to improve the reproducibility and stability of capacitive immunosensors allowing them to become a reliable option for miniaturized devices and application in the rapidly growing field of point of care diagnostic devices.

\section{Conflicts of interest}

There are no conflicts to declare.

\section{Acknowledgments}

The authors acknowledge the financial support from the Natural Science and Engineering Research Council of Canada (NSERC), the Fonds de Recherche Nature et Technologies-Team Grant, the NSERC-CREATE in ISS, and FRC scholarship from Consejo Nacional de Ciencia y Teconología de México (CONACYT) and Secretaría de Educación Pública de México (SEP). The authors will also like to thank all the staff from McGill University Nanotools-Microfab for their insights for the fabrication of the IDEs used in this work.

\section{References}

1 1. V. M. Mirsky, M. Riepl and O. S. Wolfbeis, Biosensors and Bioelectronics, 1997, 12, 977-989.

2 2. J. A. Goode, J. Rushworth and P. A. Millner, Langmuir, 2015, 31, 6267-6276.

3 3. X. Luo and J. J. Davis, Chemical Society reviews, 2013, 42, 5944-5962.

4 4. C. Berggren, B. Bjarnason and G. Johansson, Electroanalysis, 2001, 13, 173-180.

5 5. M. I. Prodromidis, Electrochim. Acta, 2010, 55, 4227-4233.

6 6. Z. Zou, J. Kai, M. J. Rust, J. Han and C. H. Ahn, Sensors and Actuators, A: Physical, 2007, 136, 518-526.

7 7. E. Ghafar-Zadeh, S. F. Chowdhury, A. Aliakbar, V. Chodavarapu, R. Lambrose, L. Beital, M. Sawan and M. Trifiro, Biomedical microdevices, 2010, 12, 967-975.

8 8. A. Qureshi, J. H. Niazi, S. Kallempudi and Y. Gurbuz, Biosensors and Bioelectronics, 2010, 25, 2318-2323.

9 9. J.-W. Park, S. Saravan Kallempudi, J. H. Niazi, Y. Gurbuz, B.S. Youn and M. B. Gu, Biosensors and Bioelectronics, 2012, 38, 233-238.

10 10. J. J. BelBruno, G. Zhang and U. J. Gibson, Sensors and Actuators B: Chemical, 2011, 155, 915-918.

11 11. C. Sontimuang, R. Suedee and F. Dickert, Anal Biochem, 2011, 410, 224-233.

12 12. X. Luo, M. Xu, C. Freeman, T. James and J. J. Davis, Anal. Chem., 2013, 85, 4129-4134.

13 13. X. L. Luo, Q. Xu, T. James and J. J. Davis, Anal. Chem., 2014, 86, 5553-5558.
14 14. B. C. Jacquot, N. Muñoz, D. W. Branch and E. C. Kan, Biosensors and Bioelectronics, 2008, 23, 1503-1511.

15 15. M. Lee, N. Zine, A. Baraket, M. Zabala, F. Campabadal, R. Caruso, M. G. Trivella, N. Jaffrezic-Renault and A. Errachid, Sensors and Actuators B: Chemical, 2012, 175, 201-207.

16 16. A. Gebbert, M. Alvarez-Icaza, W. Stoecklein and R. D. Schmid, Anal. Chem., 1992, 64, 997-1003.

17 17. M. Riepl, V. M. Mirsky, I. Novotny, V. Tvarozek, V. Rehacek and O. S. Wolfbeis, Anal. Chim. Acta, 1999, 392, 77-84.

18 18. I. Zaccari, B. G. Catchpole, S. X. Laurenson, A. G. Davies and C. Walti, Langmuir, 2014, 30, 1321-1326.

19 19. S. K. Arya, P. Zhurauski, P. Jolly, M. R. Batistuti, M. Mulato and P. Estrela, Biosensors and Bioelectronics, 2018, 102, 106112.

20 20. A. Markov, K. Greben, D. Mayer, A. Offenhäusser and R. Wördenweber, ACS Appl. Mater. Interfaces, 2016, 8, 16451 16456.

21 21. P. Sanguino, T. Monteiro, F. Marques, C. J. Dias, R. Igreja and R. Franco, IEEE Sensors Journal, 2014, 14, 1260-1265.

22 22. S. Li, H. Cui, Q. Yuan, J. Wu, A. Wadhwa, S. Eda and H. Jiang, Biosensors and Bioelectronics, 2014, 51, 437-443.

23 23. K. Kim, S. W. Park and S. S. Yang, BioChip Journal, 2010, 4, 148-154.

24 24. H. Cui, S. Li, Q. Yuan, A. Wadhwa, S. Eda, M. Chambers, R. Ashford, H. Jiang and J. Wu, Analyst, 2013, 138, 7188-7196.

25 25. J. S. Daniels and N. Pourmand, Electroanalysis, 2007, 19, 1239-1257.

26 26. Z.-S. Wu, J.-S. Li, T. Deng, M.-H. Luo, G.-L. Shen and R.-Q. Yu, Anal. Biochem., 2005, 337, 308-315.

27 27. E. B. Bahadir and M. K. Sezginturk, Artif. Cell. Nanomed. Biotechnol., 2016, 44, 248-262.

28 28. M. Labib, M. Hedstrom, M. Amin and B. Mattiasson, Anal. Bioanal. Chem., 2010, 397, 1217-1224.

29 29. E. P. Randviir and C. E. Banks, Anal. Methods, 2013, 5 1098-1115.

30 30. L. Yang, Y. Li, C. L. Griffis and M. G. Johnson, Biosensors and Bioelectronics, 2004, 19, 1139-1147.

31 31. M. Ibrahim, J. Claudel, D. Kourtiche, B. Assouar and M. Nadi, in New Developments and Applications in Sensing Technology, Springer, 2011, pp. 169-189.

32 32. D.-Y. Jeon, S. J. Park, Y. Kim, M.-J. Shin, P. S. Kang and G.T. Kim, Journal of Vacuum Science \& Technology B, 2014, 32, 021803.

33 33. V. Tsouti, C. Boutopoulos, I. Zergioti and S. Chatzandroulis, Biosensors and Bioelectronics, 2011, 27, 1-11.

34 34. M. R. Shoar Abouzari, F. Berkemeier, G. Schmitz and D. Wilmer, Solid State Ionics, 2009, 180, 922-927.

35 35. T. Pajkossy, Solid State Ionics, 2005, 176, 1997-2003.

36 36. T. Pajkossy and L. Nyikos, Physical Review B, 1990, 42, 709719.

37 37. G. A. McRae, M. A. Maguire, C. A. Jeffrey, D. A. Guzonas and C. A. Brown, App/ Surf Sci, 2002, 191, 94-105.

38 38. P. Van Gerwen, W. Laureyn, W. Laureys, G. Huyberechts, M. Op De Beeck, K. Baert, J. Suls, W. Sansen, P. Jacobs and L. Hermans, Sensors and Actuators B: Chemical, 1998, 49, 73-80.

39 39. E. Boubour and R. B. Lennox, Langmuir, 2000, 16, 42224228.

40 40. N. V. Subbarao, M. Gedda, S. Vasimalla, P. K. Iyer and D. K. Goswami, physica status solidi (a), 2014, 211, 2403-2411.

41 41. R. Nathawat, A. Kumar, N. K. Acharya and Y. K. Vijay, Surface and Coatings Technology, 2009, 203, 2600-2604.

42 42. K. V. Singh, A. M. Whited, Y. Ragineni, T. W. Barrett, J. King and R. Solanki, Anal. Bioanal. Chem., 2010, 397, 1493-1502.

43 43. B. C. Shekar, S. Sathish and R. Sengoden, Physics Procedia, 2013, 49, 145-157.

44 44. B. B. Narakathu, M. Z. Atashbar and B. E. Bejcek, Biosensors and Bioelectronics, 2010, 26, 923-928. 
